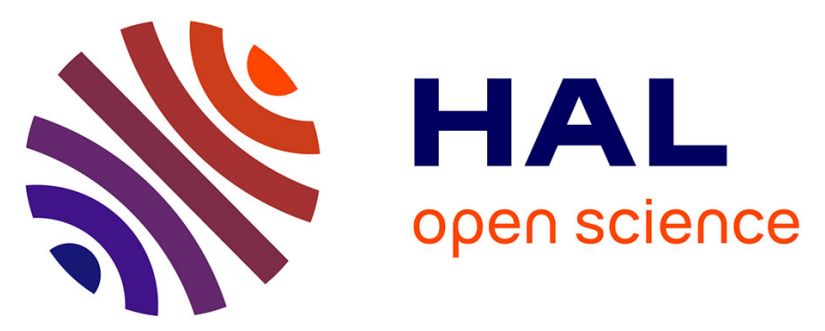

\title{
Nouvelles données sur le petit âge de glace en Corse : apports de l'analyse croisée des informations géomorphologique, palynologique et archéologique de la piève de Santo Pietro (désert de l'Agriate, Corse)
} Marc-antoine Vella, Émilie Tomas, Ghjasippina Thury-Bouvet, Serge Muller

\section{To cite this version:}

Marc-antoine Vella, Émilie Tomas, Ghjasippina Thury-Bouvet, Serge Muller. Nouvelles données sur le petit âge de glace en Corse : apports de l'analyse croisée des informations géomorphologique, palynologique et archéologique de la piève de Santo Pietro (désert de l'Agriate, Corse). Méditerranée, 2014, 122, pp.99-111. 10.4000/mediterranee.7170 . hal-03048781

\author{
HAL Id: hal-03048781 \\ https://hal.science/hal-03048781
}

Submitted on 9 Dec 2020

HAL is a multi-disciplinary open access archive for the deposit and dissemination of scientific research documents, whether they are published or not. The documents may come from teaching and research institutions in France or abroad, or from public or private research centers.
L'archive ouverte pluridisciplinaire HAL, est destinée au dépôt et à la diffusion de documents scientifiques de niveau recherche, publiés ou non, émanant des établissements d'enseignement et de recherche français ou étrangers, des laboratoires publics ou privés. 


\title{
Nouvelles données sur le petit âge de glace en Corse: apports de l'analyse croisée des informations géomorphologique, palynologique et archéologique de la piève de Santo Pietro (désert de l'Agriate, Corse)
}

\author{
New data on the Little Ice Age in Corsica: a comparison of geomorphological, palynological \\ and archaeological results from Santo Pietro (Désert de l'Agriate, Corsica)
}

\author{
Marc-Antoine VELLA \\ Science pour l'Environnement \\ UMR 6134 CNRS-Université de Corse \\ Campus Grimaldi, 20250 Corte \\ Laboratoire Sisyphe UMR 7619 \\ CNRS-Université Pierre et Marie Curie \\ 75252 Paris
}

\author{
Ghjasippina THURY-BOUVET \\ Science pour l'Environnement \\ UMR 6134 CNRS-Université de Corse \\ Campus Grimaldi, 20250 Corte
}

\author{
Serge MULLER \\ Université Montpellier 2 \\ serge.muller@univ-montp2.fr
}

\begin{abstract}
Résumé - Les modifications climatiques et les activités anthropiques influencent l'évolution des versants depuis l'Holocène récent. L'étude des documents fiscaux de la piève de Santo Pietro au cours des $X V^{e}$ et $\mathrm{XVI} \mathrm{I}^{\mathrm{e}}$ siècles renseigne sur la position des sites médiévaux et les dynamiques de la population. Les documents cartographiques anciens (1776-1850) et récents (2010) permettent de restituer l'organisation du territoire et de caractériser les ensembles géomorphologiques. La synthèse de l'analyse sédimentaire de trois sondages associée à l'étude palynologique du vallon de Spizicciu permet de préciser les modifications des paysages au cours de l'Anomalie Climatique Médiévale ( 750-1000 AD) et du petit âge glaciaire ( 1450-1850 AD). Nous observons une augmentation des processus d'érosion/sédimentation de certains secteurs des vallées corses à partir de $\sim 1150$ cal. AD en relation avec des changements de végétation. L'histoire de l'occupation humaine de l'île ainsi que celle des techniques agraires mises en place à cette époque peuvent être ainsi reliées à une modification importante du paysage.
\end{abstract}

Mots clés: géomorphologie, palynologie, géoarchéologie, Petit Âge de Glace, Corse

La géoarchéologie des îles méditerranéennes s'est développée au cours des années 1990 et a permis de mettre en évidence plusieurs changements environnementaux majeurs depuis la seconde moitié de l'Holocène. En Corse, peu de recherches permettent de retrouver ces principaux évènements. Les travaux réalisés au cours des années 80-90 sur le remplissage pollinique des étangs côtiers et les lacs d'altitude, mettent en évidence le rôle prépondérant des sociétés sur l'évolution de la composition de la végétation (fig. 1). La position géostratégique de la Corse au sein de la Méditerranée fait de cette île un lieu de contact entre les civilisations de la région. L'évolution technique et culturelle des sociétés insulaires est bien connue en comparaison des changements environnementaux. Au début du XIr ${ }^{\mathrm{e}}$ siècle, l'île est divisée en cinq évêchés, héritage des circonscriptions de l'époque paléochrétienne. Ces diocèses sont eux-mêmes fractionnés en soixante-dix secteurs, appelés pièves (ISTRIA et al., 2013).

La Corse présente une importante diversité de contextes géomorphologiques dont les caractéristiques évoluent différemment en fonction de l'intensité de l'occupation humaine et des changements climatiques.
Abstract - Climate changes and human activities have influenced the evolution of valley slopes during the late Holocene. The study of 15 th and 16 th century tax documents from Santo Pietro's Piève provides information on both the position of medieval sites and population dynamics. Ancient (1776-1850) and recent maps (2010) allow the territorial geography to be reconstructed and to characterize the geomorphology at the micro-regional scale. Survey work, chronostratigraphy and palynological studies of the Spizicciu valley allow us to probe landscape changes during the Medieval Climate Anomaly ( 750-1000 AD) and the Little Ice Age ( 1450$1850 \mathrm{AD}$ ). We observe an increase in erosion / sedimentation rates in Corsican valleys from $\sim 1150$ cal. AD related to vegetation changes. The history of human occupation of the island, in addition to the farming techniques implemented at this time, can be related to significant changes in the landscape.

Keywords: geomorphology, palynology, geoarchaeology, Little Ice Age, Corsica

Les modifications du paysage consécutives des pratiques agropastorales sont encore trop peu étudiées et les adaptations des sociétés aux changements climatiques ne sont pas clairement identifiées en Corse. Les données paléoenvironnementales récentes acquises pour le Désert de l'Agriate ont permis de restituer l'évolution des versants depuis la fin du IV $\mathrm{IV}^{\mathrm{e}}$ millénaire $\mathrm{BC}$ et mettent en évidence une augmentation de la sédimentation à partir du XII ${ }^{e}$ siècle (VELLA et al., 2013).

L'approche que nous avons privilégiée pour cette étude vise à mettre en évidence les interactions entre l'homme et son environnement au cours du Moyen Âge. Grâce à une méthode pluridisciplinaire associant l'analyse sédimentologique de trois sondages réalisés dans trois secteurs géographiques différents de la piève de Santo Pietro. L'apport de données sur la composition de la végétation est issu de l'étude palynologique d'un de ces sondages. Cette synthèse permet de retracer et de déterminer la part de l'influence du climat et de l'anthropisation sur l'évolution des versants et de proposer une base de discussion sur les modalités du PAG en contexte insulaire méditerranéen. 


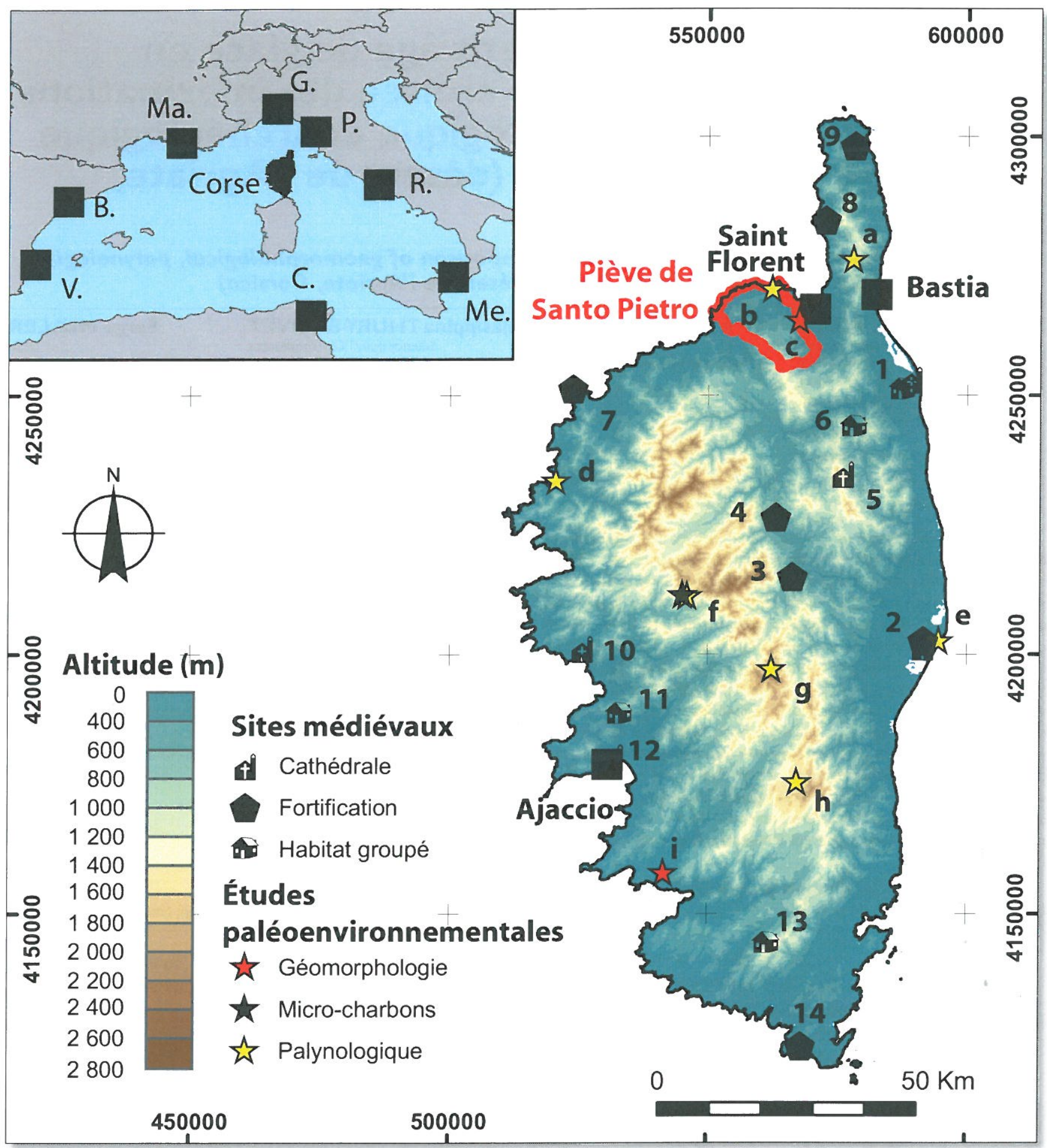

Fig. 1 - Carte de situation (B.: Barcelone; C.: Carthage; G.: Gênes; Me.: Messine; Mr.: Marseille; P.: Pise; R. Rome; V.: Valence). Principaux sites médiévaux. 1: Mariana; 2: Aleria; 3: Corte; 4: Rostino, 5: Accia; 6: Cariolu; 7: Calvi; 8: Nonza; 9: San Colombanu; 10: Sagone; 11: Muntichji; 12: Ajaccio; 13: Ortolo; 14: Bonifacio. Principales études paléoenvironnementales. a: Bocca Antillo; b: Saleccia; c: Monte Revincu; d: Le Fango; e: Étang del Sale; f: Lac de Creno; g: Lac de Bastani; h: Plateau du Coscione; i: Marais de Canniccia.

\section{I - Contexte général}

\section{I.I - Contexte géomorphologique et géologique}

Le désert de l'Agriate est un secteur de basse montagne localisé dans le nord de la Corse. Le paysage est composé de collines séparées par les principaux cours d'eau: le Liscu $\left(13,317 \mathrm{~km} ; 37,112 \mathrm{~km}^{2}\right)$ le Fiume Santu $(11,719 \mathrm{~km}$; $\left.19,623 \mathrm{~km}^{2}\right)$ et le Purette $\left(10,969 \mathrm{~km} ; 15,254 \mathrm{~km}^{2}\right)$. Cette zone géographique est délimitée au sud par le massif du Tenda qui culmine à plus de $1500 \mathrm{~m}$, à l'est par la vallée de l'Aliso, au nord par une côte rocheuse et à l'ouest par la vallée du Liscu. Les formations géologiques qui composent le substrat de ce secteur sont principalement composées de granitoïdes (Carbonifère supérieur), de formations volcaniques et volcano-sédimentaires (Permien) à l'ouest et d'ensembles géologiques issus de la croute océanique (Jurassique sup.) à l'est (ROSSI et al., 2001) (fig. 2). Les données sur le PAG en Corse sont lacunaires ou inexistantes et la topographie de l'île induit une forte diversité de paysages.

Les études récentes menées sur le contexte géomorphologique de la partie orientale du Désert de 
l'Agriate ont permis d'identifier différents évènements détritiques depuis la seconde moitié de l'holocène (VELLA, 2010). Deux sondages ont été réalisés dans ce secteur. Le premier, MRE 01, réalisé dans le vallon de Spizicciu, a livré la plus longue séquence chronostratigraphique (1,90 $\mathrm{m}$ de profondeur). Le second, MR 03 (0,90 $\mathrm{m}$ de profondeur) a enregistré la dynamique sédimentaire du vallon de Purette. Ces travaux montrent que les processus sédimentaires sont particulièrement importants depuis le $\mathrm{XII}^{\mathrm{e}}$ siècle. Les informations que nous présentons dans cet article visent à synthétiser ces résultats pour les périodes Médiévale et Moderne. De plus, l'apport de données sur le remplissage pollinique du sondage MRE 01 permet de mettre en relation l'évolution sédimentaire des versants avec celle de la végétation micro-régionale.

Le plateau de Capo Castinco (altitude moyenne: $472 \mathrm{~m} \mathrm{NGF}$ ) est une vaste étendue d'environ 300 hectares comprenant de légers reliefs et comptant quelques fontaines disséminées, plusieurs ruisseaux saisonniers ou

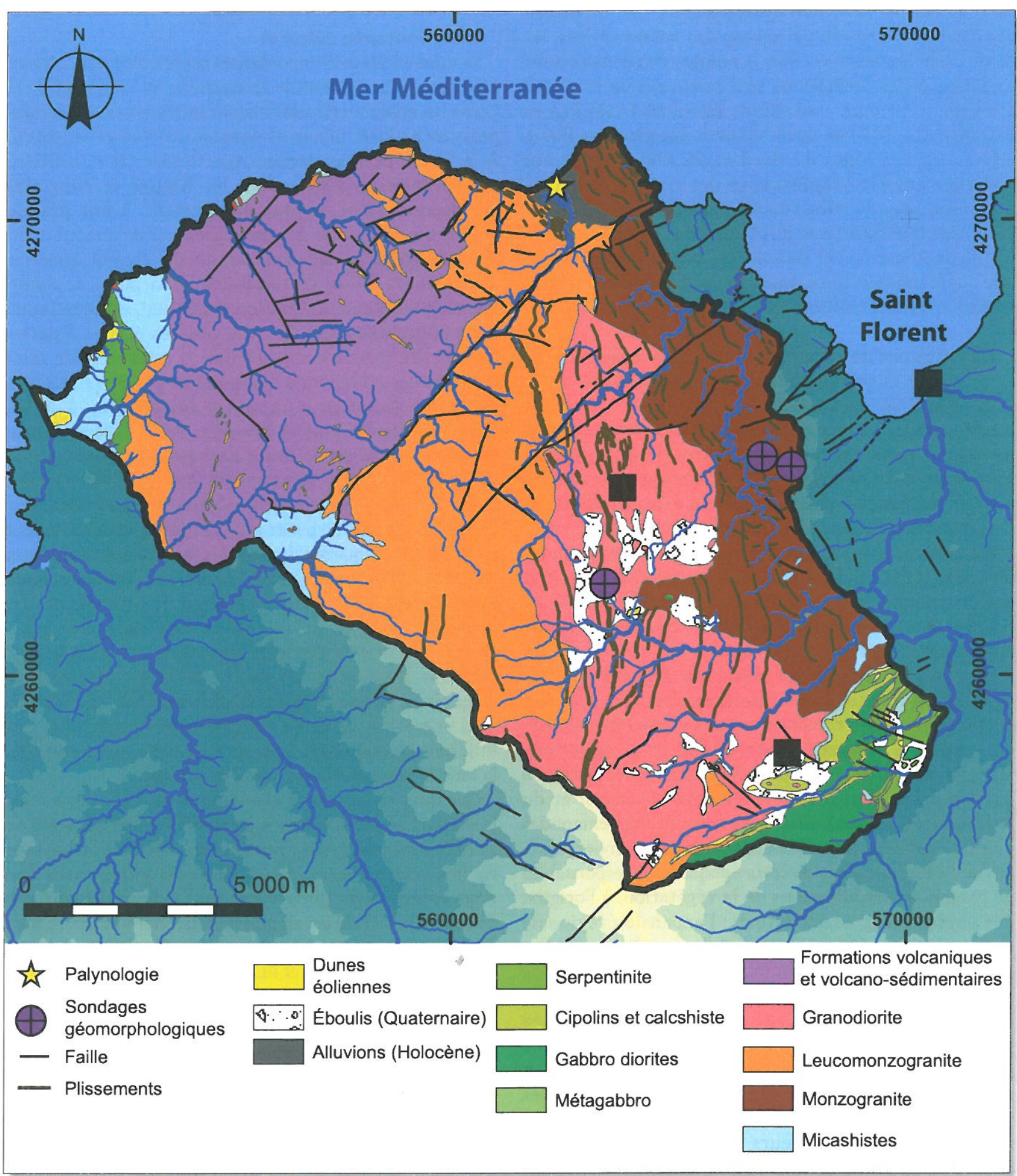

Fig. 2 - Carte géologique simplifiée de la piève de Santo Pietro. D'après Rossi et al., 2001. 
permanents et une zone humide dans sa partie centrale. Ce plateau est dominé au sud par le Monte Filetto ( $842 \mathrm{~m}$ ) et au nord par les reliefs de Mamucci $(573 \mathrm{~m}$ ) dont le col se dégage nettement dans le paysage. Le sondage MAM 2 (1,10 $\mathrm{m}$ de profondeur) été réalisé en arrière d'un mur de contention des terres sur le versant sud du col de Mamucci dont l'âge de mise en place n'est pas clairement identifié.

À une échelle régionale, les études géomorphologiques sur les îles de la Méditerranée et sur le pourtour méditerranéen montent que l'anomalie climatique médiévale (ACM), située entre 750-1000 apr. J.-C., est caractérisée par un déficit hydrologique et par un niveau bas des lacs d'altitude. Au niveau des basses vallées, les cours d'eau tendent à réduire le nombre de chenaux ainsi qu'à inciser les formations sédimentaires de la plaine alluviale (CAROZZA et al., 2011; ARNAUD-FASSETTA et PROVANSAL, 1999). Au cours du petit âge glaciaire (PAG), les vallées des Alpes du Nord et du Rhône montrent une augmentation significative des crues à partir du $\mathrm{XIV}^{\mathrm{e}}$ siècle jusqu'au milieu du XIX ${ }^{\mathrm{e}}$ (LEROY LADURIE, 1983; BRAVARD, 1989; JORDA et RODITIS, 1994). De même, sur la plaine du Roussillon et du Lez, les cours d'eau montrent une augmentation importante de la sédimentation dans les basses vallées (JORDA, 2000 ; CAROZZA et al., 2013).

\section{I.2 - Apport des données paléo-écologiques pour la compréhension des modalités du PAG en Corse}

Les secteurs que nous avons étudiés sont situés en basse montagne, dans des vallons de petits ruisseaux ou sur un plateau de basse altitude. La végétation de ce secteur est représentée par une composition caractéristique de l'étage mésoméditerranéen (AMANDIER et al, 1982).

Les études sur le remplissage en micro-charbons du lac de Creno $(1310 \mathrm{~m}$ ) dans le massif du Rotondo montrent une diminution du signal incendie à partir de $1600 \mathrm{cal}$. BP (LEYS et al., 2013). En revanche, l'influence du PAG n'est pas clairement identifiée.

L'analyse du remplissage pollinique de l'étang côtier de Saleccia montre le rôle prédominant des activités anthropiques sur la composition de la végétation au cours de la période antique, du Moyen Âge et de la période Moderne (fig. 1 et 2). La période romaine est marquée par l'introduction probable du noyer (Juglans) et du châtaignier (Castanea), ainsi que par le développement des cultures de chêne liège (Quercus suber) et de l'olivier (Olea). Les activités humaines de cette époque sont responsables de profondes modifications de la végétation, à l'origine des paysages régionaux actuels: l'expansion régionale du pin maritime (Pinus pinaster) et l'apparition locale des éléments thermophiles du maquis (Myrtus, Selaginella). Au cours du XIV ${ }^{\text {e }}$ siècle, l'exploitation des forêts lors de l'occupation génoise entraîne une dégradation généralisée des ensembles forestiers (REILLE, 1992). En 1584, le gouverneur de Gêne impose la culture du châtaignier, de l'olivier, du figuier et du mûrier à proximité des habitations. Cette composition de la végétation va être conservée pendant plusieurs siècles et permettre une certaine prospérité pour les villages de la Castaniccia, dans l'est de la Corse. Entre 1774 et 2013, une régression des châtaigneraies de l'est de la Corse est accompagnée d'une augmentation des surfaces forestières. Cette évolution est à relier à une diminution de la population humaine dans les secteurs d'étude (SAN ROMAN SANZ et al., 2013). L'analyse des pollens de la séquence sédimentaire du vallon de Spizicciu permet de renseigner l'évolution de la végétation à une échelle micro-régionale. Le croisement avec les données sédimentaires acquises sur ce vallon, le vallon de Purette, ainsi que sur le plateau de Capo Castinco permet de retracer l'évolution du paysage au cours du PAG.

\section{I.3 - Contexte culturel}

La mise en place d'importantes routes commerciales en Méditerranée occidentale au cours du XI ${ }^{\text {e }}$ siècle fait de la Corse un enjeu territorial majeur entre les trois grandes puissances Pise, Gênes et Aragon (ISTRIA et al., 2013). À la fin du premier Moyen Âge, le territoire insulaire est délimité en pièves dont les limites territoriales correspondraient, approximativement, à une grande vallée ou du moins à une région relativement bien délimitée géographiquement par des crêtes montagneuses et des cours d'eau. Le territoire de la piève de Santo Pietro, qui s'étend sur environ $176,10 \mathrm{~km}^{2}$, est mentionné pour la première fois dans les sources écrites à partir de 1114 (fig. 3). Cette documentation nous informe aussi de l'existence d'habitats non fortifiés nés avant le début du XII ${ }^{e}$ siècle autour d'un centre domanial ou d'unités d'exploitation (TOMAS et PEREIRA, 2012). C'est au niveau de cette entité structurale de base que s'applique le prélèvement fiscal seigneurial. Aux XII ${ }^{\mathrm{e}} \mathrm{XIII}^{\mathrm{e}}$ siècles, le peuplement se structure d'habitats établi à mi-hauteur de pente composé de petits groupes de maisons, rarement fortifiés. Pratiquement tous les habitats connus aux XII $^{e}$-XIII ${ }^{e}$ siècles apparaissent encore dans les documents écrits des $\mathrm{XV}^{\mathrm{e}}$ et $\mathrm{XVI}^{\mathrm{e}}$ siècles. Il s'agit de petits hameaux, organisés autour de la cellule familiale d'origine. L'image de la villa des XIII $^{\mathrm{e}}-\mathrm{XIV}^{\mathrm{e}}$ siècles contraste avec celle que donnent les textes des $\mathrm{XV}^{\mathrm{e}}$ et $\mathrm{XVI}^{\mathrm{e}}$ siècles dans lesquels ce terme désigne le plus souvent une unique agglomération de taille relativement importante. Ces différences conduisent à penser qu'à la fin du Moyen Âge, la terminologie est floue, voire incertaine.

Quelques villages sont abandonnés au XVI ${ }^{\mathrm{e}}$ siècle mais la plupart se maintiennent à travers les siècles, voire se développent puisque cet habitat est à l'origine du paysage de la Corse moderne. Les structures villageoises que nous connaissons aujourd'hui sont le fruit d'évolution, de mutations et de recomposition de l'habitat qui se sont développés majoritairement au cours des derniers siècles du Moyen Âge (TOMAS et PEREIRA, 2012).

\section{2 - Méthodes d'étude}

Afin de mettre en évidence les modifications paléoenvironnementales et leurs interactions avec les sociétés médiévales de l'île au cours du PAG, nous avons opté pour une approche interdisciplinaire centrée sur le secteur géographique de la piève de Santo Pietro. 


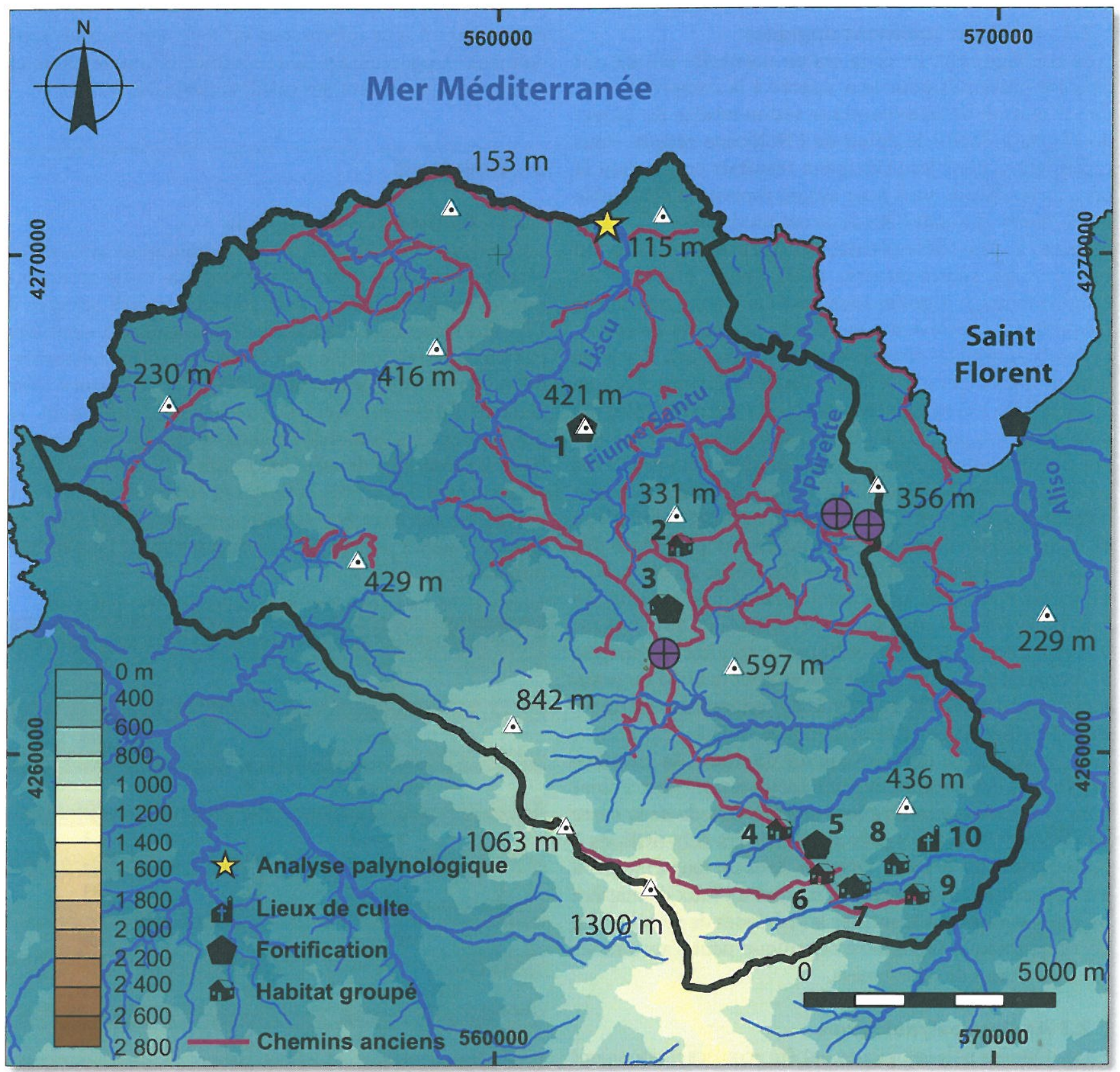

Fig. 3 - Localisation des sites médiévaux de la Piève de Santo Pietro. 1: Montaggione; 2: Casta; 3 : Torricella; 4: Forcioni; 5 : Brumica; 6 : Catarello; 7: Zuccaraccia; 8: Casenove; 9: Junchelli; 10: Santo Pietro.

\section{I - Cartographie et SIG}

Les documents fiscaux des xve et xvie siècles sont, sans nul doute, les plus riches en information sur l'évolution et la répartition de la population de la piève. Même si certains établissements ne sont pas systématiquement notifiés dans les tailles, cette documentation offre un aperçu de la démographie de la piève de 1454 à 1537.

Le Plan Terrier, réalisé entre 1776 et 1778, nous informe sur le réseau viaire, la localisation des communaútés, la nature des productions agricoles ainsi que de la localisation des zones de maquis. L'exploitation des données de ce document reste limitée en raison de l'emplacement approximatif des sites indiqués et de l'absence du parcellaire.

Les cadastres napoléoniens (1800-1850) et actuels ont permis de vérifier l'occupation pérenne de plusieurs sites médiévaux. Pour les habitats abandonnés définitivement, la toponymie, si elle est conservée, permet de localiser l'emplacement d'un site pour lequel aucun vestige ne subsiste sur le terrain. Cela doit être réalisé avec précaution car un toponyme peut souvent être utilisé à plusieurs endroits du territoire.

L'analyse des cartes IGN, des cartes géologiques, des photographies aériennes permettent d'apporter de nouvelles informations qui permettent de caractériser les différents ensembles géomorphologiques mais aussi d'identifier les marqueurs de la mobilité des paysages.

Les informations issues des documents fiscaux et cartographiques ont été vectorisées et géoréférencées grâce à un SIG leur permettant ainsi d'être mises en relation et visualisées. L'apport de cette étude a permis de localiser les différents sites datant du Moyen Age et de l'époque Moderne, d'identifier les réseaux de chemins mais aussi de réaliser la carte géomorphologique de la Piève de Santo Pietro. 


\section{2 - Analyses sédimentologiques}

Le sondage MRE 01 et la coupe naturelle MR 03 ont déjà été présentés pour leur apport à la compréhension de l'évolution de la dynamique sédimentaire du Désert de l'Agriate depuis le début de l'Holocène récent. Nous rappellerons donc les principaux résultats concernant la période médiévale sans pour autant reprendre l'ensemble des données issues de l'étude de ces sondages.

Suite à une détermination stratigraphique du remplissage sédimentaire, nous avons effectué un échantillonnage régulier tous les $5 \mathrm{~cm}$ en augmentant la fréquence de prélèvements aux changements de facies. Certains ensembles sédimentaires ont livré des charbons qui ont fait l'objet d'une détermination anthracologique avant d'être envoyé au laboratoire de Poznań (Pologne) pour datation par ${ }^{14} \mathrm{C}$. Au total, sept datations AMS ont été ainsi réalisées (tableau 1). Les résultats ont ensuite été calibrés grâce au logiciel Calib 6.0 en utilisant la courbe de calibration Intcal09.14c (REIMER et al., 2009). En raison de la nature des sédiments, nous avons privilégié des mesures de la granulométrie par voie sèche de la fraction sableuse. Pour cela, nous avons utilisé une colonne de tamis aux mailles: 0,$063 ; 0,08 ; 0,1 ; 0,12 ; 0,15 ; 0,2 ; 0,25$; 0,$315 ; 0,5 ; 0,63 ; 0,8 ; 1,1,25$ et $2 \mathrm{~mm}$. Le résultat de l'analyse sédimentologique permet de déterminer la taille du grain moyen et ainsi de différencier la dynamique de transport et d'identifier les conditions de dépôt.

Concernant la détermination du taux de matière organique, l'ensemble des échantillons ont fait l'objet d'un protocole opératoire basé sur la méthode de la perte au feu (DEAN, 1974). Après avoir mis les échantillons à l'étuve, ces derniers sont chauffés dans un four à moufle à $450^{\circ} \mathrm{C}$ pendant cinq heures. La mesure du taux de matière organique permet d'identifier les périodes de stabilité des versants lorsque la matière organique peut s'accumuler lors des phases de pédogenèse.

\subsection{Analyse palynologique}

Les données palynologiques ont été acquises uniquement sur le sondage MRE 01. Des prélèvements de sédiments ont été réalisés sur coupe en raison de l'assèchement estival, et de la compaction très importante des sédiments qui rend le carottage difficile. Des volumes de $1 \mathrm{~cm}^{3}$ de sédiment ont été prélevés à intervalles plus ou moins réguliers (entre 2 et $5 \mathrm{~cm}$ ) le long du profil sédimentaire, et soumis à des bains d'acide dans le but d'éliminer la silice, la cellulose et les carbonates. Le résidu, très riche en pollen $\left(1 \mathrm{~cm}^{3}\right.$ de sédiment contient entre 10000 et 1000000 de grains de pollen), est enfin analysé sous microscope optique. Les courbes sont données en pourcentage de la somme pollinique totale et les points représentent des valeurs inférieures à $1 \%$.

\section{3 - Résultats}

\section{I - Analyse cartographique}

L'analyse des documents cartographiques anciens et récents associée à celle des registres de taille montrent que les sites médiévaux sont souvent encore occupés à l'époque moderne. La tendance démographique, identifiée par les registres de taille, agit de deux manières opposées: décroissance pour les secteurs côtiers et croissance pour ceux de l'intérieur. Avec la poussée du peuplement, la physionomie des formes de l'habitat ne change pas. Certains habitats sont plus densément peuplés, ils s'agrandissent et absorbent les unités cellulaires environnantes. Pour compléter ces données démographiques, il convient de signaler que six petites ville ont disparu entre 1454 et 1537 (tableau 2).

Tableau 2 - Population de la piève de Santo Pietro en 1454 et 1537 AD

\begin{tabular}{|c|c|c|}
\hline Piève de Santo Pietro & $1454 \mathrm{AD}$ & 1537AD \\
\hline Monte & 12,5 & 7 \\
\hline Petragie & 7 & 11 \\
\hline Zuccaraccia & 2 & - \\
\hline Catarello & 11 & 26 \\
\hline Casenove & 9 & 15 \\
\hline Caseluche & 8 & - \\
\hline Junchelli & 22 & - \\
\hline Fornace & 5 & 8 \\
\hline San Gavino & 22,5 & 26 \\
\hline Guezzi & 37 & 41 \\
\hline Pogio & 8 & - \\
\hline Vitallaccie & 37 & 63 \\
\hline Pianello & 13 & 24 \\
\hline Caseboniasche & 3 & 4 \\
\hline Vigna & 2 & - \\
\hline Lumito & 16 & 29 \\
\hline Pogiolo & 6,5 & - \\
\hline Cabio & 5 & - \\
\hline TOTAL & 226,5 & 268 \\
\hline
\end{tabular}

Le paysage est connoté, entre autre, par son aspect, son relief ou sa fonction. Le territoire de la piève de Santo Pietro présente des caractéristiques physiques qui permettent de comprendre l'organisation des sociétés anciennes. Le réseau de chemin et les villages ont pu être inclus dans le SIG. La toponymie montre aussi plusieurs allusions à des

Tableau 1 - Datation réalisées.

\begin{tabular}{|c|c|c|c|c|c|c|c|}
\hline Nom de l'échantillon & $\mathbf{N}^{\circ}$ US & $\begin{array}{l}\text { Profondeur } \\
\text { (en cm) }\end{array}$ & $\begin{array}{c}\text { Type } \\
\text { de matériel }\end{array}$ & $\begin{array}{l}\text { Code } \\
\text { laboratoire }\end{array}$ & $\begin{array}{c}\text { Âge } \\
\text { cal. BP }\end{array}$ & Erreur \pm & Age cal. BC/AD 20 \\
\hline MAM 2 & 4 & 63 & $\begin{array}{c}\text { Charbon } \\
\text { (Cistaceae / Erica) }\end{array}$ & POZ 35429 & 530 & 30 & $1320-1440 A D$ \\
\hline MRE $0140 \mathrm{~cm}$ & 7 & 40 & Charbon & POZ 35416 & 480 & 30 & $1407-1453 \mathrm{AD}$ \\
\hline MRE $0155 \mathrm{~cm}$ & 9 & 55 & Charbon & POZ 14026 & 940 & 30 & $1020-1160 A D$ \\
\hline MRE $0190 \mathrm{~cm}$ & 14 & 90 & Charbon & POZ 35418 & 3345 & 35 & $1736-1527 \mathrm{BC}$ \\
\hline MRE 03 G $15-25 \mathrm{~cm}$ & 2 & $15-25$ & Charbon & POZ 35424 & 75 & 30 & $1691-1925 A D$ \\
\hline MRE $03 \mathrm{G} 45-50 \mathrm{~cm}$ & 5 & $45-50$ & Charbon & POZ 35425 & 185 & 30 & $1650-1955 A D$ \\
\hline MRE $03 \mathrm{D} 56 \mathrm{~cm}$ & 3 & 56 & Charbon & POZ 35426 & 900 & 30 & $1040-1211 A D$ \\
\hline
\end{tabular}




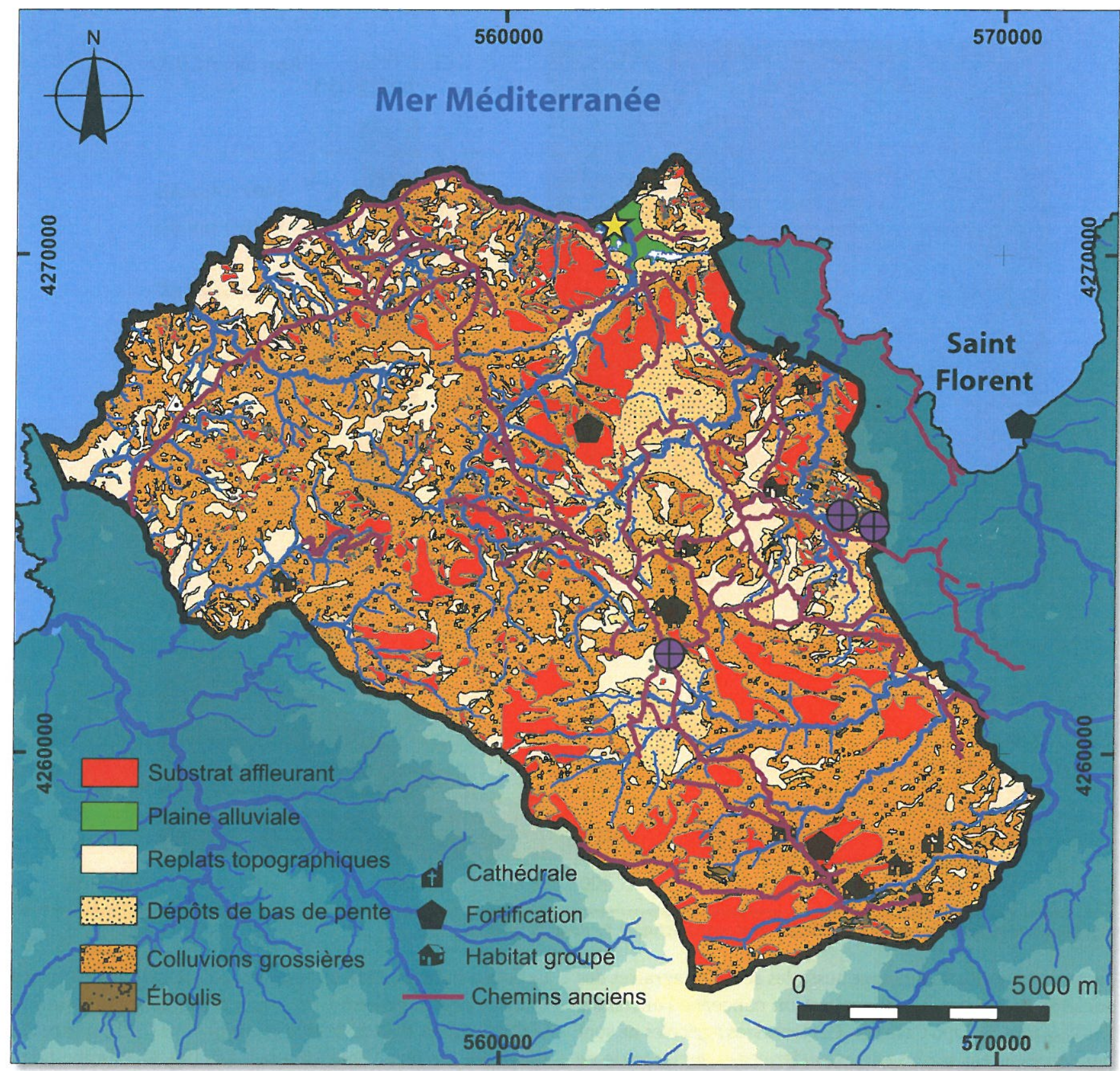

Fig. 4 - Carte géomorphologique de la Piève de Santo Pietro.

pratiques agricoles et pastorales, à des sources ainsi qu'à des secteurs de mares temporaires. Ainsi, Le toponyme Vetricia est un indice supplémentaire de l'existence d'une ou plusieurs mares temporaires au niveau du plateau de Capo Castinco pour les périodes anciennes.

Les documents cartographiques récents montrent que le Désert de l'Agriate est composé d'une mosaïque d'ensemble géomorphologiques (fig. 4). Nous pouvons ainsi différencier trois ensembles plus ou moins continus qui sont caractéristiques de la couverture sédimentaire superficielle des versants:

- une zone où le substrat est affleurant où sub-affleurant, incultivable, forme un ensemble presque continu du Monte Genova au Monte Revincu. Cette barrière topographique isole cette région du reste du désert de l'Agriate. Cet ensemble correspond aussi aux zones d'érosion, ou le ruissellement de surface est maximum.
- les plaines alluviales sont relativement restreintes dans la piève de Santo Pietro.

- une troisième zone correspond aux dépôts de pente. Ces formations sédimentaires regroupent les colluvions plus ou moins grossières et les éboulis. Les replats topographiques sont aussi inclus dans cet ensemble bien que leur couverture sédimentaire soit principalement issue de l'arénisation des granitoïdes.

La plaine de Casta et de Teddi forment un ensemble où les dépôts de bas de pente et les replats topographiques sont pratiquement continus. Le plateau de Capo Castinco est bien individualisé au sein de la piève de Santo Pietro. Il est caractérisé par des colluvions grossières à fines avec une zone humide en son centre. La position du site médiéval de Torricella est clairement en relation avec ces caractéristiques géomorphologiques. La concentration importante de 


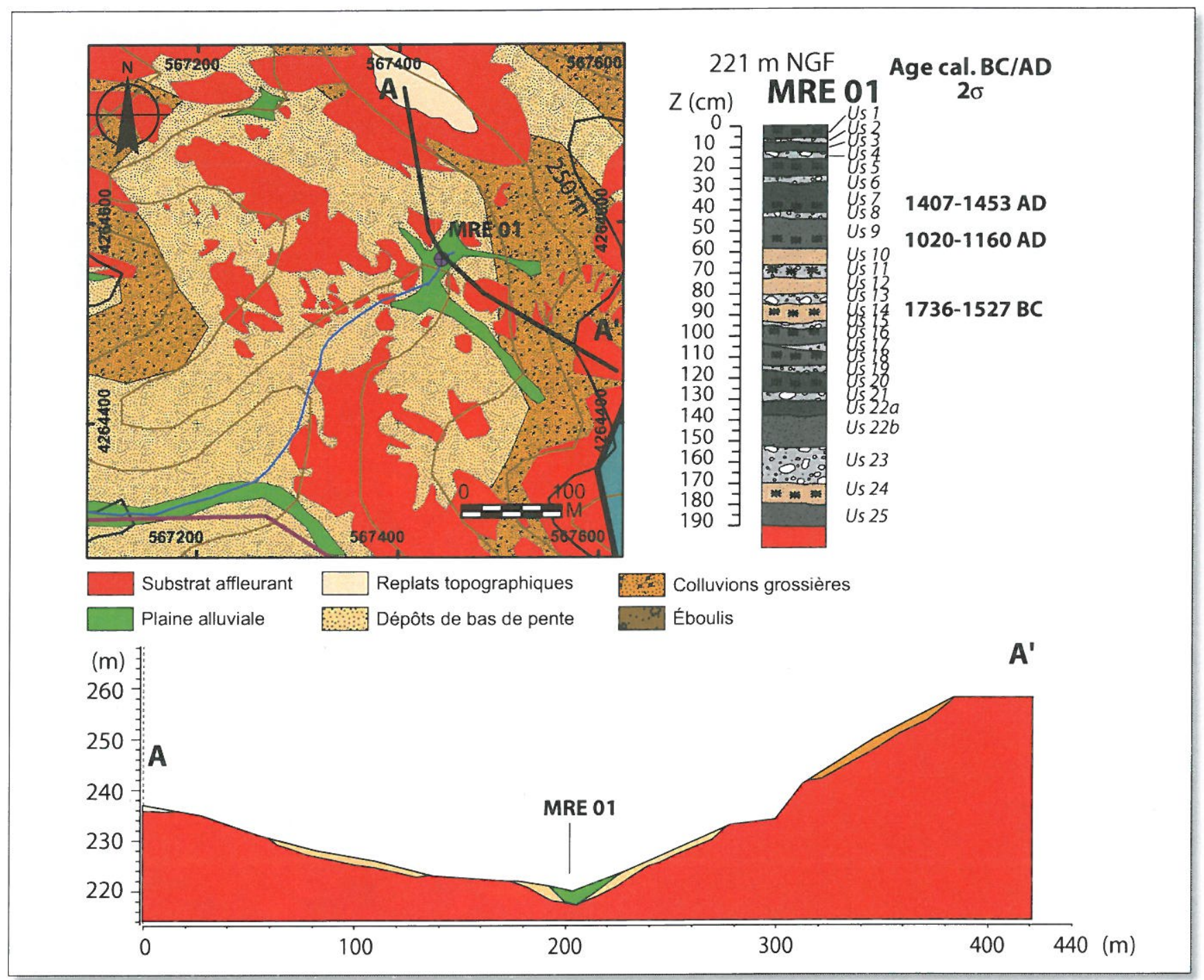

Fig. 5 - Synthèse des données géomorphologiques et stratigraphiques du vallon de Spizicciu.

terrasses de culture témoigne aussi des activités agraires des sociétés anciennes. Leur localisation concerne l'ensemble des versants de Capo Castinco. Ces terrasses agricoles ne sont pas représentées sur les cartes anciennes et aucun texte ne permet de déterminer l'origine de leur construction.

\section{2 - Résultats de l'analyse stratigraphique et sédimentologique}

Les données stratigraphiques et sédimentaires des sondages MRE 01 et MR 03 ont déjà été présentées plus en détail (VELLA et al., 2014). Les résultats montrent que la stratigraphie du vallon de Spizicciu et de Purette est composée d'une alternance hétérométrique de niveaux fins et de niveaux grossiers (fig. 5). Cette organisation montre de fortes variations de la dynamique sédimentaire. Concernant les dépôts contemporains de l'époque médiévale, les formations sédimentaires sont caractéristiques de dépôts alluviaux. L'apport de datations ${ }^{14} \mathrm{C}$, réalisées dans différentes unités sédimentaires (Us), a permis de situer l'augmentation de la sédimentation autour de de 1020-1160 cal. AD. Plusieurs phases d'apports détritiques marquent ensuite la stratigraphie du vallon. Ainsi, vers 1407-1453 cal. AD un nouvel apport détritique peut être identifié. Les apports ultérieurs du vallon de Spizicciu n'ont pu être datés.
La coupe naturelle MR 03 réalisée dans le vallon de Purette montre une augmentation importante de la dynamique sédimentaire depuis le début du premier millénaire de notre ère environ (1040-1211 cal. AD) (fig. 6). Comme pour le sondage MRE 01, plusieurs phases de dépôt ont été identifiées dans la première moitié du XIX ${ }^{e}$ siècle.

Le sondage MAM 2 a permis d'explorer la terrasse inférieure du versant sud du relief de Mamucci (fig. 7). Au-dessus du substrat rocheux granitique (R) qui a été atteint à plus d'1 $\mathrm{m}$ de profondeur, une stratification complexe a enregistré six Us. L'Us 1 est caractérisée par une texture sablo-limoneuse. Le second ensemble sédimentaire (Us 2) est constitué d'éléments relativement plus grossiers. Le troisième ensemble (Us 3), de granulométrie plus fine, a livré des charbons millimétriques dont la datation a donné des âges situés autour de 1320-1440 cal AD. La quatrième Us est composée d'éléments plus grossiers issus de la remobilisation des sédiments du versant.

\section{3 - Résultat de l'analyse palynologique du vallon de Spizicciu}

Les données palynologiques sont conservées malgré l'acidité du sédiment (fig. 8). Les données palynologiques nous renseignent sur la composition végétale microrégionale comme nous l'indique le fort 


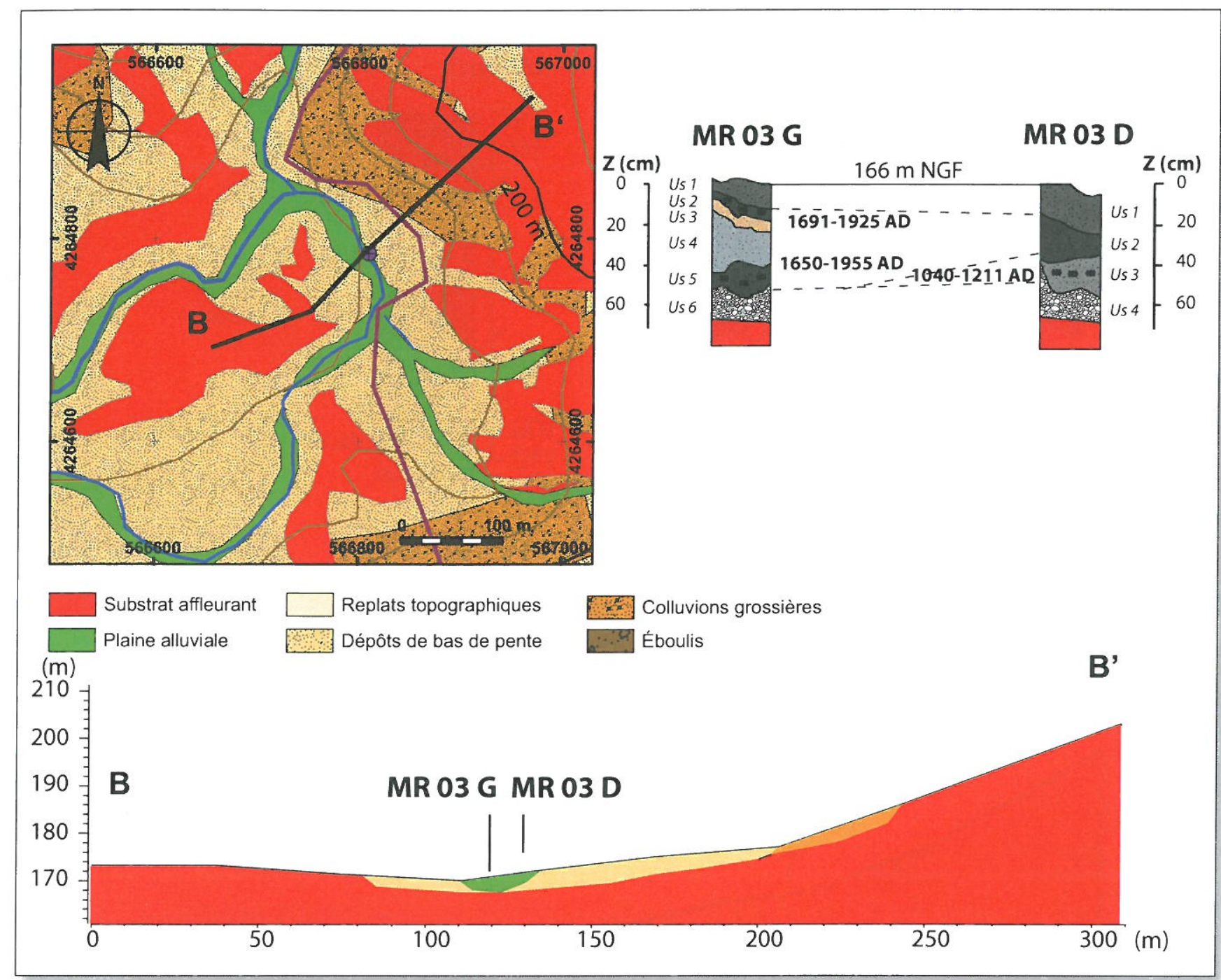

Fig. 6 - Synthèse des données géomorphologiques et stratigraphiques du vallon de Purette.

taux de bruyère arborescente et le faible taux de pins. L'environnement végétal est clairement dominé par des forêts de bruyère arborescente et où les arbres ne tiennent qu'une place très restreinte. L'évolution des pourcentages de certaines espèces nous intéresse plus particulièrement.

La présence tardive de pollen de céréales vers $70 \mathrm{~cm}$ (avant 1020-1160 A.D) est à relier aux problèmes de conservation des pollens, à la part forcément restreinte des céréales dans l'assemblage végétal environnant et à la provenance locale des pollens. Ces remarques laissent envisager que l'agriculture dans ce secteur pourrait être plus ancienne. Le chêne vert est noté en simple présence avec toutefois une augmentation lors des périodes modernes. La dominance de bruyère arborescente est sujette à des variations. La baisse brutale de son taux de pollens à partir de 1020-1160 cal. AD et l'augmentation synchrone des asteroideae et des cistes indiquent une ouverture du milieu et sa colonisation par des espèces de reconquête. Les Isoètes sont très intéressants pour la reconstruction paléoenvironnementale du désert de l'Agriate. En effet, elle est spécifique d'un environnement restreint, limitée aux fonds de vallons périodiquement ennoyés et formant des zones humides de l'automne au printemps. L'augmentation progressive de ces taux de pollens indique un développement de la zone.

\section{4 - Comparaisons du remplissage sédimentaire des trois secteurs pour la période concernant le PAG}

Les analyses sédimentaires menées sur la piève de Santo Pietro permettent de restituer la dynamique sédimentaire des versants de la partie orientale du désert de l'Agriate depuis le premier millénaire de notre ère. Entre $~ 1600$ cal BC et $\sim 1150$ cal. AD, les processus sédimentaires identifiés dans le vallon de Spizicciu sont caractérisés par une diminution des apports détritiques. Le vallon de Purette et le versant nord du plateau de Capo Castinco n'ont pas livré d'éléments de datation permettant de déterminer la répartition spatiale des secteurs avec une faible dynamique sédimentaire. Dans le vallon de Spizicciu, les formations sédimentaires sont caractérisées par une texture proche de celles de formations alluviales et sont datées de 1020-1160 cal. AD. Le Purette, qui comprend le vallon de Spizicciu, présente une accumulation sédimentaire sableuse bien triée sur des formations issues de l'arénisation des granitoïdes. Les charbons retrouvés au sein de l'ensemble sableux ont permis de dater cet apport détritique du début du premier millénaire AD (1040-1211 cal. AD). Les différents secteurs que nous avons étudiés montrent donc une augmentation significative des processus sédimentaires vers $1150 \mathrm{cal}$. AD. 


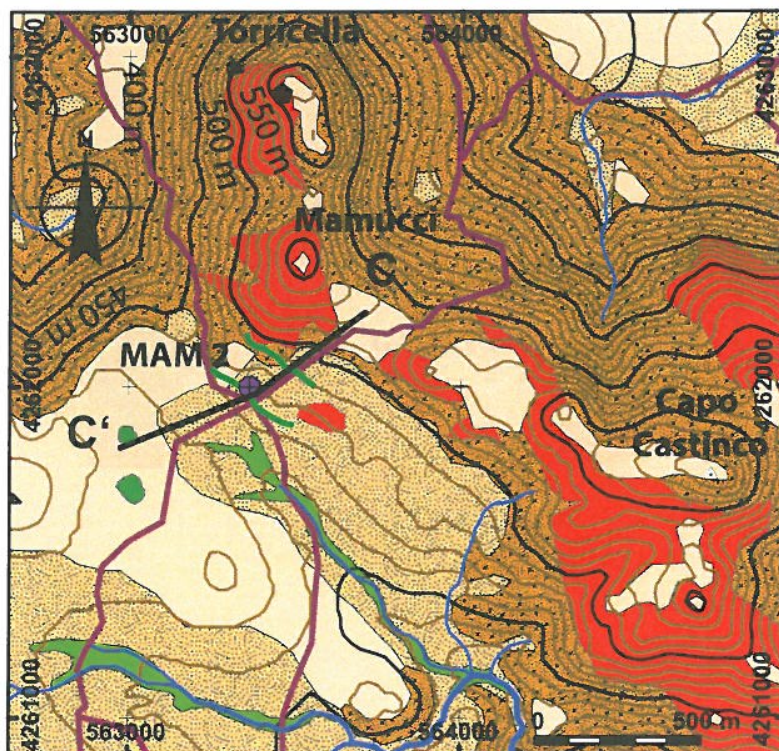

\section{$Z(\mathrm{~cm})$}

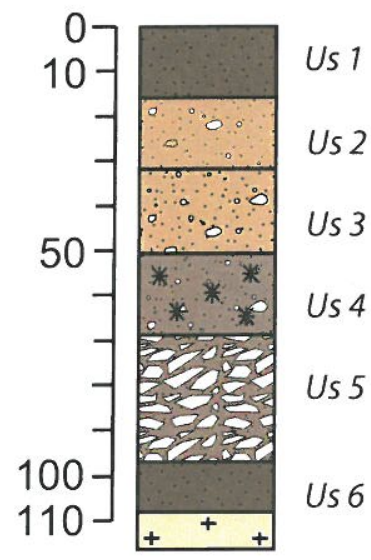

1320

1440 cal AD

Us 6

Colluvions grossières

Éboulis

(m)

540 - C

MAM 2

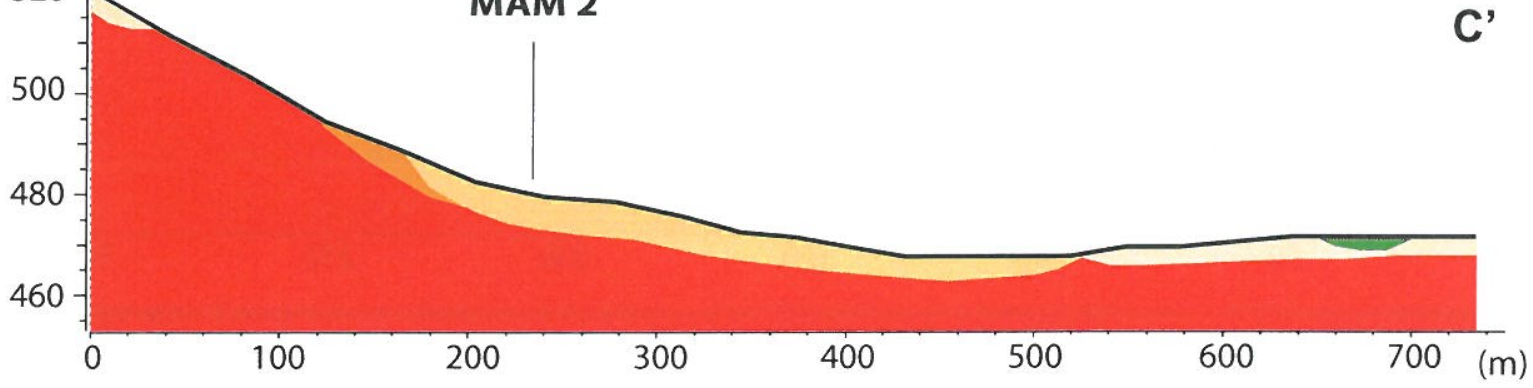

Fig. 7 - Synthèse des données géomorphologiques et stratigraphiques du plateau de Capo Castinco

À partir de cette période, plusieurs phases détritiques peuvent être individualisées selon les secteurs. Les formations sédimentaires et archéologiques piégées en arrière des structures de contention des terres du plateau de Capo Castinco montrent une augmentation de l'épaisseur de dépôts à partir de $\sim 1380$ cal. AD. Cependant, l'existence d'un niveau de destruction de la terrasse de culture suggère que l'aménagement initial du versant pourrait provenir d'une période plus ancienne. Le vallon de Spizicciu montre une nouvelle phase d'apports détritiques vers $1430 \mathrm{cal}$. AD. Le manque de datations pour les niveaux sédimentaires supérieurs du vallon de Spizicciu ne permet pas de restituer précisément la chronologie des évènements. Cependant, deux niveaux grossiers attestent de phases d'augmentation de la dynamique sédimentaire. Ces dernières peuvent être retrouvées dans le vallon de Purette et sont situées vers le début du XIX ${ }^{e}$ siècle.

\section{5 - Évolution du paysage de la piève de Santo Pietro au cours du PAG}

Les données sur la composition de la végétation croisées avec l'analyse de la dynamique sédimentaire des versants de la partie orientale du désert de l'Agriate permettent de restituer l'évolution du paysage depuis le début du premier millénaire $\mathrm{AD}$. Les taxons marqueurs de l'anthropisation sont particulièrement bien représentés à partir de 1150 cal. AD. Cette mise en culture des versants s'accompagne de défrichements importants de la forêt de bruyère arborescente par le feu entre $\sim 1150$ et $\sim 1800$ cal. AD comme l'attestent le développement des formations de ciste.

L'augmentation des processus détritiques qui commencent autour de 1150 cal. AD est synchrone du début de l'ouverture de la forêt de bruyère arborescente. Cette évolution progressive est clairement à relier aux activités agropastorales des sociétés médiévales. Les taxons représentatifs de l'évolution du milieu de dépôts montrent qu'entre $\sim 1150$ et $\sim 1800$ cal. AD, le vallon de Spizicciu devait être en eau durant la majeure partie de l'année. Les formations sédimentaires mises en place au cours de cette période indiquent une augmentation des processus détritiques et attestent ainsi d'une modification du paysage. Enfin, à partir du début du $\mathrm{XIX}^{\mathrm{e}}$ siècle, l'augmentation de la représentation des arbres dans l'assemblage de la végétation témoigne 


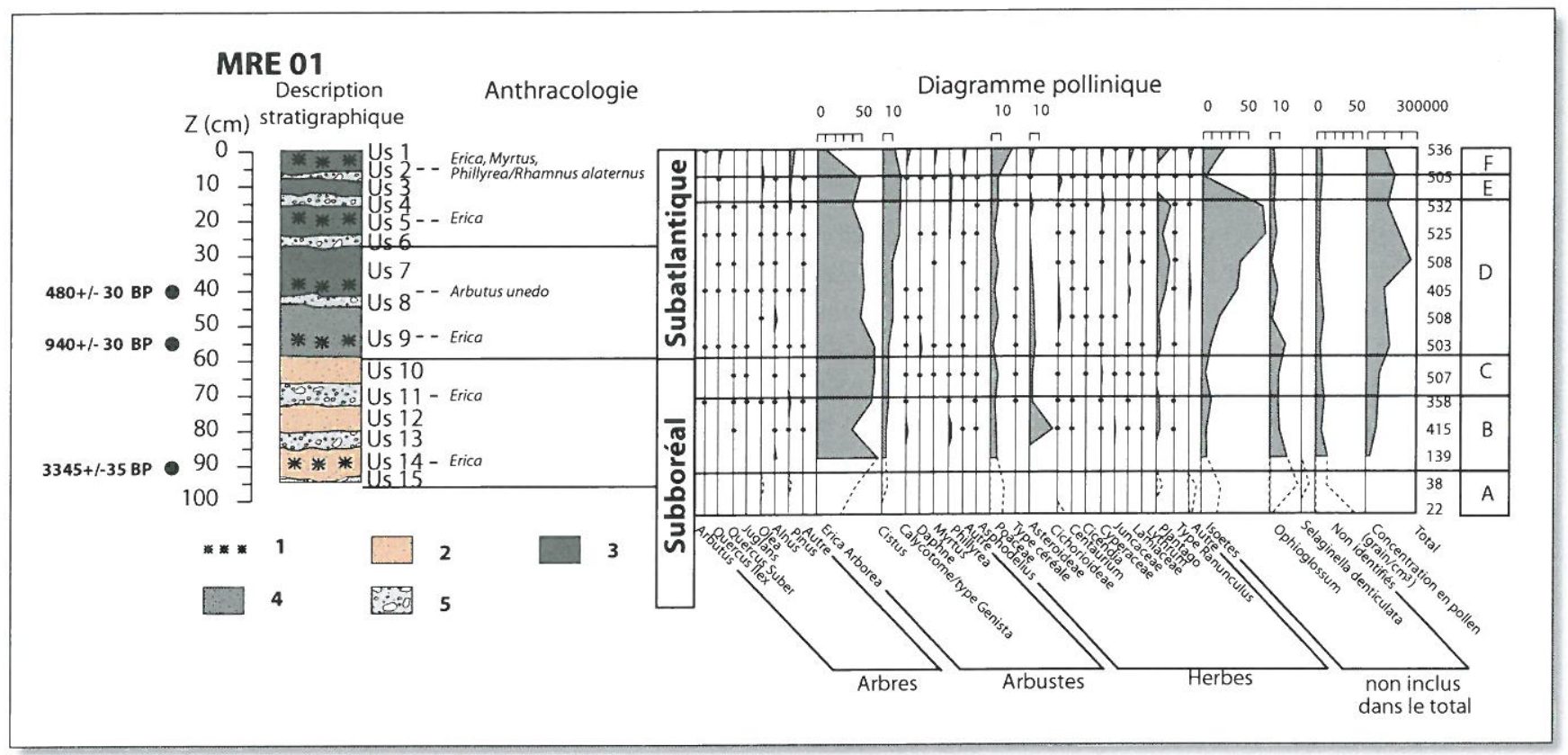

Fig. 8 - Résultat de l'analyse pollinique du vallon de Spizicciu.

d'une déprise agricole. Cependant, l'aspect actuel de la végétation témoigne d'une influence importante des feux, naturels et anthropiques, qui conduit à une morphologie caractéristique d'un maquis bas.

\section{6 - La part de l'influence climatique et de l'influence anthropique sur l'évolution du paysage au cours du PAG.}

Le PAG en Méditerranée est caractérisé par une augmentation des processus sédimentaires entre le XIV ${ }^{e}$ et le XIX ${ }^{e}$ siècle. Si cette augmentation est clairement d'origine climatique, les phénomènes observés dépendent aussi de l'intensité des aménagements anthropiques. En Corse, Le territoire de la piève de Santo Pietro présente un morcellement géomorphologique que les sociétés médiévales ont su exploiter et contrôler. Les derniers siècles du second Moyen Âge voient la mise en place lente et progressive d'une société villageoise qui était déjà en marche en Europe du Sud depuis le XI ${ }^{\mathrm{e}}$ siècle (TOMAS et PEREIRA, 2012). Historiquement, la décroissance démographique est causée par les phénomènes liés au paludisme et aux invasions qui provoquent souvent l'abandon des habitats (ISTRIA et al., 2013). Dans les secteurs côtiers, l'habitat, composé d'une multitude de petits noyaux plus ou moins concentrés, est remodelé à la fin du Moyen Âge. À cause des guerres, des attaques barbaresques répétées, les pièves sont progressivement dévastées. Les principales zones d'habitat de la piève sont concentrées au niveau de l'actuel village de Santo Pietro di Tenda. Cette position, à mi-hauteur de pente et sur un replat topographique, permet de contrôler l'accès au plateau de Capo Castinco et à la plaine de Casta-Teddi depuis la vallée de l'Aliso. Les tours de Montaggione et de Torricella devaient aussi permettre la surveillance d'une partie du terroir. L'augmentation des processus hydrosédimentaires dans le Désert de l'Agriate entre le XII et le XIX ${ }^{e}$ siècle peut avoir contraint les sociétés à entreprendre des travaux d'aménagement des versants dans le secteur de Capo Castinco mais aussi probablement dans d'autres secteurs du nord de la Corse. Ces travaux attestent ainsi d'une connaissance des potentialités agricoles des terres ainsi que d'une perception du risque que constitue l'érosion des sols sur les potentialités agricole des terroirs. L'évolution du paysage de la piève de Santo Pietro au cours du PAG est donc principalement consécutive des activités agropastorales. En revanche l'intensité des dépôts sédimentaires entre le milieu du $\mathrm{XII}^{\mathrm{e}}$ siècle et le XIX ${ }^{\mathrm{e}}$ siècle semble reliée à une augmentation des épisodes hydrologiques dont la fréquence ne peut être déterminée pour l'instant. Les études sur l'évolution des paysages méditerranéens au cours de l'anomalie climatique médiévale (ACM, 750-1000 AD) et du petit age glaciaire (PAG, 1350-1850 AD) montrent une augmentation des processus sédimentaires qui peuvent être à l'origine d'une modification importante de la position et de l'organisation de l'habitat (VELLA et al., 2013; CAROZZA et al., 2011; ARNAUD-FASSETTA et PROVANSAL, 1999; JORDA et RODITIS, 1994; BRAVARD, 1989). En Corse, la succession de périodes sèches et humides au cours du PAG a pu favoriser la formation et le développement d'étangs en arrière de cordon littoraux sableux. La présence de ces zones humides est clairement en relation avec la prolifération des moustiques et pourrait être à l'origine des épidémies de paludisme au cours du Moyen Âge.

\section{Conclusion}

Les recherches paléoenvironnementales que nous avons menées sur la piève de Santo Pietro permettent donc de mettre en évidence l'augmentation des processus détritiques qui a été identifiée en Europe au cours entre le $\mathrm{XIV}^{\mathrm{e}}$ et le XIX ${ }^{e}$ siècle. L'organisation de la piève est à relier aux contraintes physiques du territoire mais aussi à celles 
des incursions barbaresques du Moyen Âge. La Corse est marquée depuis le $\mathrm{V}^{\mathrm{e}}$ millénaire $\mathrm{BC}$ par d'importantes modifications du paysage en relation avec l'occupation humaine de l'île. La déstabilisation climatique du PAG, qui est principalement importante en Corse au cours des XII et le $\mathrm{XIII}^{\mathrm{e}}$ siècles et à la fin du XVIII ${ }^{\mathrm{e}}$ siècle, conduit les populations de ce secteur à entretenir les terres les plus fertiles grâce à la construction de terrasses de culture. Le développement des zones humides conduit aussi à l'augmentation des épidémies de paludisme. L'organisation du territoire corse actuel, qui provient en grande partie de la fin du Moyen Âge, est donc le fruit de l'évolution climatique mais aussi des relations entre les civilisations de Méditerranée occidentale. La réalisation de sondages dans les basses vallées de l'Agriate permettra d'affiner la chronologie des événements et de restituer plus précisément la mobilité des paysages au cours du PAG. De même, la fouille des sites médiévaux et notamment Torricella, permettra de mieux comprendre les activités domestiques et agropastorales des populations de ce secteur et ainsi de mieux appréhender les relations que les sociétés médiévales insulaires ont tissés avec leur environnement.

\section{Remerciements}

Nous tenons à remercier les deux relecteurs anonymes, les équipes de prospections de surface ainsi que les fouilleurs bénévoles. Nous remercions aussi F. Leandri, C. Gilabert et C. Jorda pour nous avoir permis de mener ces recherches ainsi que la DRAC de Corse pour le financement de nos travaux.

\section{Bibliographie}

AMANDIER L., DUREAU R., LAURENT J.-L., JOFFRE L.-M., JOFFRE R., (1982), Eléments pour un zonage agro-sylvo-pastoral de la Corse, ministère de l'Agriculture, région Corse, $78 \mathrm{p}$.

ARNAUD-FASSETTA G., PROVANSAL M., (1999), High frequency variations of water flux and sediment discharge during little ice age in the Rhone delta (France). Relationship to the catchment area, Developments in Hydrobiology, 410, p. 241-250.

BRAVARD J.-P., (1989), La métamorphose des rivières des alpes françaises à la fin du Moyen Âge et à l'époque moderne, Liège, Rev. Geogr, 25, p. 145-157.

CAROZZA J.-M., PUIG C., ODIOT T., VALETTE P., PASSARIUS O., (2011), Lower Mediterranean plain accelerated evolution during the Little Ice Age: geoarchaeological insight in the Tech basin (Roussillon, Gulf of lion, Western Mediterranean), Quaternary international, 30, 1, p. 1-11.

CAROZZA J.-M, PUIG C., ODIOT T., PASSARRIUS O., VALETTE P., (2013), L'édification de la Basse Plaine de la Salanque (Roussillon, France) au cours de la seconde partie de l'holocène et ses implications sur la répartition des sites archéologiques. Quaternaire, 24, 2, p. 155-165.

DEAN W.E. Jr., (1974), Determination on carbonate and organic matter in calcareous sediments and sedimentary rocks by loss on ignition: comparison with other methods, Journal of Sedimentary Petrology, 44, p. 242-248.

ISTRIA D., COLOMBANI P., CORBARA A.-G., DURAND A., FRANZINI A. PEREIRA E., TOMAS E., USAÏ N., CESARI J., (2013), Le Moyen Âge en Corse, Centre régional de documentation pédagogique de Corse, $84 \mathrm{p}$.

JORDA C., (2000), Morphogenèse alluviale et aménagements dans la plaine deltaïque du Lez depuis 6000 ans: la fouille archéologique de sauvetage de Port-Ariane (Lattes, Hérault), Méditerranée, 1-2, p. 25-32.

JORDA M., RODITIS J.-C., (1994), Les épisodes de gel sur le Rhône depuis l'An Mil. Périodisation, fréquence, interprétation climatique, Méditerranée, 3-4, p. 19-30.

LEROY LADURIE E. (1983), Histoire du climat depuis l'An Mil, deux volumes, Paris, Flammarion.

LEYS B., CARCAILLET C., DEZILEAU L., ALI A. A., BRADSHAW R. H. W., (2013), A comparison of charcoal measurements for reconstruction of Mediterranean paleo-fire frequency in the mountains of Corsica. Quaternary research, 79, p. 337-349.

REILLE M., (1992), New pollen-analytical researches in Corsica: the problem of Quercus ilex L. and Erica arborea L., the origin of Pinus halepensis Miller forests, New Phytologist, 122, p. 359-378.

REIMER P.J., BAILLIE M.G.L., BARD E, BAYLISS A., BECK J.W., BLACKWELL P.G., RAMSEY B., BUCK CE, BURR GS, EDWARDS RL, FRIEDRICH M, GROOTES P.M., GUILDERSON T.P., HAJDAS I., HEATON T.J., HOGG A.G., HUGHEN K.A., KAISER K.F., KROMER B., MCCORMAC F.G., MANNING S.W., REIMER R.W., RICHARDS D.A., SOUTHON J.R., TALAMO S., TURNEY C.S.M., VAN DER PLICHT J., WEYHENMEYER C.E., (2009), IntCal09 and Marine09 radiocarbon age calibration curves, 0-50,000 years cal BP, Radiocarbon, 51, 4, p. 1111-1150.

ROSSI PH., DURAND-DELGA M., LAHONDÈRE J.-C, BAUD J.-P., EGAL E., LAHONDÈRE D., LAPORTE D., LLUCH D., LOŸE-PILOT M.-D., OHNENENSTETTER M., PALAGI P., (2001), Carte géologique France (1/50000), feuille Santo-Pietro-di-Tenda (1106). Orléans : BRGM. Notice explicative par Rossi Ph., Durand-Delga M., Lahondère J.-C., Lahondère D., 224 p.

SAN ROMAN SANZ A., FERNANDEZ C., MOUILLOT F., FERRAT L., ISTRIA D., PASQUALINI V., (2013), Long-Term Forest Dynamics and Land-Use. Abandonment in the Mediterranean Mountains, Corsica, France. Ecology and Society, 18, 2, 38. en ligne: [http://dx.doi. org/10.5751/ES-05556-180238].

TOMAS E., PEREIRA E., (2012), Bilan des recherches archéologiques de l'habitat du château de Rostino, Bulletin de la Société des Sciences et d'Histoire de la Corse, 734-735, p. 7-25.

VELLA M.-A., (2010), Approches géomorphologique et géophysique des interactions sociétés/milieux en Corse au cours de l'Holocène. Thèse, Université de Corse, 2 volumes, $324 \mathrm{p}$. 
VELLA M.-A., GHILARDI M., DIOUF O, PARISOT J.-C., HERMITTE D., PROVANSAL M., FLEURY J., DUSSOUILLEZ P., DELANGHE-SABATIER D., DEMORY F., QUESNEL Y., ANDREAS HARTMANN-VIRNICH A., DELPEY Y., BERTHELOT M., BICKET A., (2013), Géoarchéologie du Rhône dans le secteur du pont Saint-Bénézet (Avignon, Provence, France) au cours de la seconde moitié du deuxième millénaire apr. J.-C.: étude croisée de géographie historique et des paléoenvironnements, Géomorphologie: relief, processus, environnement, 3, p. $287-310$.

VELLA M.-A., LEANDRI F., JORDA C., GILABERT C., OTTAVIANI-SPELLA M.-M., TABBAGH A., (2014), Reconstitution de la sédimentation au cours de l'Holocène récent en contexte archéologique dans le massif du Monte Revincu (Santo Pietro di Tenda, Corse): l'apport de l'approche géoarchéologique, Quaternaire, à paraître. 